\title{
FORTUNA Y TRAYECTORIA ESCÉNICA DE UNA COMEDIA CALDERONIANA: BIEN VENGAS, MAL, SI VIENES SOLO ${ }^{1}$
}

\author{
Rafael González Cañal \\ Departamento de Filología Hispánica y Clásica \\ Facultad de Letras \\ Universidad de Castilla-La Mancha \\ Avda. Camilo José Cela, s/n \\ 13071 Ciudad Real. España \\ Rafael.GCanal@uclm.es
}

[Anuario calderoniano (ISSN: 1888-8046), 3, 2010, pp. 137-153]

Poco sabemos de la fecha de composición de la comedia calderoniana Bien vengas mal, si vienes solo. Tenemos datos sobre su estreno que tuvo lugar en palacio el 16 de diciembre de 1635 por la compañía de Tomás Fernández Cabredo. Cruzada Villaamil omite este nombre con lo que indujo a error a Rennert y Cotarelo que pensaron que la obra había sido representada por la compañía de Martínez de los Ríos, nombre que aparecía en la entrada precedente ${ }^{2}$. Shergold y Varey co-

${ }^{1}$ Este artículo se incluye dentro de los proyectos FFI2008-05884-C04-01/FILO y CSD2009-00033 (Consolíder), aprobados por el Ministerio de Ciencia e Innovación.

2 Cruzada Villaamil, 1871; Cotarelo, 1924, p. 168; Rennert, 1906-1907. 
rrigieron posteriormente este error ${ }^{3}$. La misma compañía de Tomás Fernández, representó días después también en palacio, el 13 de enero de 1636, la obra titulada El jardín de Falerina, escrita en colaboración por Rojas Zorrilla, Antonio Coello y Calderón de la Barca.

La comedia titulada Bien vengas, mal, si vienes solo consta en la lista que preparó Calderón para el duque de Veragua, que, como se sabe, se publicó por primera vez en el Obelisco fúnebre, pyrámide funesto que construía a la inmortal memoria de D. Pedro Calderón de la Barca [...] D. Gaspar Agustín de Lara (Madrid, Eugenio Rodríguez, 1684)4. Sin embargo, no consta en la lista confeccionada por Calderón para don Francisco Marañón, aunque puede tratarse simplemente de un error ${ }^{5}$.

Se conserva un manuscrito incompleto en la Biblioteca Nacional de España ${ }^{6}$, que consigna el nombre de Antonio de Escamilla, con lo que cabe pensar que le perteneció en algún momento. Sin embargo, el dramatis personae, como muy advierte Cotarelo, registra los nombres de otro elenco, que identifica, sin dar más datos, como el de la compañía de Roque de Figueroa: ${ }^{7}$

Don Luis
Guzmán, criado
Don Juan de Lara
Don Diego de Silva
Espinel
Don Bernardo, viejo
Doña Ana
Doña María
Inés, criada
Juana

Felipe Domínguez

Saluador [Jaime Salvador]

Jazimo [Jacinto Varela]

Castro

Cosme [Cosme Pérez]

Autor [Roque de Figueroa]

Señora Ynés de Yta [Inés de Hita]

Señora Ysauel de Góngora

Señora Jusepha [Josefa Lobaco]

Señora Mariana [Mariana de Olivares]

${ }^{3}$ Shergold y Varey, 1961, p. 276.

${ }^{4}$ Puede consultarse más fácilmente en Hartzenbusch, 1848, pp. xl-xlii.

${ }^{5}$ Esta lista fue publicada por Wilson, 1962.

${ }^{6}$ Madrid, BNE, ms. 15633, 45 h. (falta la tercera jornada). Se observan dos letras diferentes.

7 «Esta copia es posterior, como se ve por el nombre de Escamilla; pero el reparto es el primitivo" (Cotarelo, 1924, p. 168, nota). No obstante, no parece estar probada esta identificación que hace Cotarelo. Shergold y Varey (1961, p. 277) no señalan que se trate de la compañía de Roque de Figueroa. Tampoco se acepta esta identificación en el DICAT, 2008, "Antonio de Escamilla». 
La obra la incluyó Vera Tassis, tras la muerte de Calderón, en la Novena parte de comedias del celebre poeta español don Pedro Calderón de la Barca... Madrid, Francisco Sanz, 1691, pp. 471-513 . En el prólogo Vera Tassis señala lo siguiente:

La de Bien vengas mal dije en el Primer Tomo, que no era de don Pedro, a causa de haber visto otra con el mismo título, y registrando esta que ahora te presento, reconozco por lo artificioso de la traza y la naturaleza del verso que es legítimo parto suyo (AL LECTOR, h. $4 \mathrm{r})$.

Como se sabe, las nueve partes de Calderón tuvieron distintas reediciones en el siglo XVIII. El gran éxito de la colección preparada por Juan de Vera Tassis y Villarroel, publicada entre 1685 y 1691, provocó su reedición y además la publicación de volúmenes facticios en los que se agrupaban las correspondientes comedias de cada parte, por medio de ediciones sueltas, reproduciendo la misma portada original. Así, está comedia aparece en la novena parte de esta serie denominada Pseudo Vera Tassis9.

En el siglo XVIII, además de aparecer en la colección de Apontes en 1760 y en el Theatro Hespañol de García de la Huerta en 1785, la comedia se difundió en seis ediciones sueltas: S. 1., s. i., s. a.;Valladolid, Alonso del Riego, s. a.; Sevilla, Viuda de Francisco de Leefdael, s. a.; Sevilla, José Padrino, s. a.; Barcelona, Francisco Suriá y Burgada, s. a.; y Barcelona, Carlos Sapera, $1766^{10}$.

\section{UN EJEMPLO DE COMEDIA DE ENREDO CALDERONIANA}

Estamos ante un ejemplo acabado de lo que es la comedia de enredo de Calderón. El dramaturgo madrileño ya había escrito para entonces algunos de los ejemplos señeros del género como La dama duende o Casa con dos puertas mala es de guardar, ambas escritas en 1629.

${ }^{8}$ Para los ejemplares de estas partes (las originales, las publicadas por Vera Tassis, y las reimpresiones) y las bibliotecas en donde se conservan, ver Reichenberger, 1979, I, pp. 24-25 y 30-31.

${ }^{9}$ Ver, por ejemplo, los volúmenes R-11345-53 y T-8581-89 de la Biblioteca Nacional de España.

${ }^{10}$ Ver Reichenberger, 1979, I, pp. 155-157. En el XIX se publica en la edición de Keil (1830) y en la de Hartzenbush (1850). 
Hay que situarse, pues, en los primeros años de la década de los treinta, la época más fecunda de Calderón en cuanto a la composición de comedias. En dichas fechas escribe obras como Mañanas de abril y mayo (1632-1633), No hay burlas con el amor (1635), El escondido y la tapada (1636), etc. Bien vengas, mal, si vienes solo fue escrita probablemente en 1635 y se puede relacionar fácilmente con otra comedia titulada También hay duelo en las damas, que, a su vez también tiene puntos en común, como ya advirtió Serralta, con Amparar al enemigo, de Antonio Solís ${ }^{11}$. Ya señalaba este crítico la existencia de un fondo común de lances y resortes dramáticos de los que echan mano los dramaturgos de la época. El hispanista francés termina su artículo sugiriendo la posibilidad de que la fecha de composición de También hay duelo... sea cercana a la de Bien vengas, mal,... y no tan tardía como se creía hasta la ahora. En efecto, Hilborn apunta como fecha de También hay duelo... 1648-1650, por criterios métricos, con lo que estaríamos ante una comedia escrita bastantes años después de Bien vengas mal. La obra se conserva en un manuscrito del siglo XVII en la BNE (ms. 16939, 64 h.) y se incluyó en la Tercera parte calderoniana de 1664, fols. $247 \mathrm{r}-72 \mathrm{v}^{12}$. Por la semejanza entre ambas es factible pensar en una fecha de composición cercana, como ocurre con otras comedias «gemelas» de este tipo como La dama duende y Casa con dos puertas..., o Mañanas de abril y mayo y No hay burlas con el amor, con lo que habría que adelantar la fecha de También hay duelo... que hasta se venía barajando por la crítica.

Volviendo a Bien vengas mal..., el enredo gira entorno al resorte dramático del honor, tema fundamental en el universo dramático de Calderón. Las leyes del honor y de la hospitalidad a las que están obli-

11 Serralta, 1987. Esta obra de Solís fue representada en Sevilla en 1642 por la compañía de Manuel Vallejo e impresa en la Parte 43 de comedias de diferentes autores, en Zaragoza en 1650.

${ }^{12}$ Es verdad que el dramatis personae es muy parecido al de Cada uno para sí (coinciden los nombres de los criados Simón e Inés, de las damas, Leonor y Violante, y de uno de los galanes, D. Félix) y esta obra es necesariamente posterior a 1652 por una alusión a la rendición de Barcelona que se produjo en octubre de de dicho año. Pero también es semejante al de Bien vengas mal... con quien comparte también los nombres de la criada Inés y de uno de los galanes, D. Juan, con lo que no parece un argumento convincente para proponer una u otra fecha de composición. Además, Calderón utiliza a menudo el nombre de Inés para las criadas (ver Reichenberger, 2009, IV, p. 347). También se llama así la criada de Amparar al enemigo de Solís. 
gados los personajes generan una intriga que se va complicando progresivamente. El desenlace es el acostumbrado final feliz, con la presencia del llamado "galán suelto» ${ }^{13}$, D. Luis, que no logra su objetivo y se conforma finalmente con el rechazo de su dama.

Por tanto, nos hallamos ante una construcción pentagonal del enredo como en otras muchas comedias de Calderón y de otros dramaturgos de la época. Hay tres galanes, de los cuales uno será el «galán suelto» y dos damas. La acción se circunscribe a las casas de las dos damas y a los escarceos generalmente nocturnos en la calle y la reja.

El enredo gira en torno al secreto que debe mantener doña Ana. $\mathrm{Su}$ amiga doña María le confia el retrato de su pretendiente y, a pesar de ser descubierto en sus manos por parte de su amado, doña Ana se mantendrá fiel a su amiga y no revelará ni la identidad del caballero ni la procedencia del retrato. Por una parte, tenemos el motivo del retrato, tan frecuente en las comedias áureas, pero lo que se pone en cuestión es la obligación de las damas frente a un secreto que pone en peligro su propio honor. Así se lo hace saber el celoso don Diego al final de la primera jornada:

Diego Mujer eres, poco importa

que descubras un secreto.

No aspires, doña Ana, a ser

el prodigio destos tiempos ${ }^{14}$.

Sin embargo, la astuta doña Ana guarda el secreto y termina convenciendo a su amante de que lo ha hecho por su bien, en un diálogo lleno de matices y sutilezas: «que quien te calló el secreto / es quien más te supo amar» ${ }^{15}$. Más tarde, es capaz de resolver la complicada situación optando sabiamente:

ANA

(Si suceden

dos daños, es el menor

el que ha de elegirse siempre ${ }^{16}$.)

13 Serralta, 1988.

14 Calderón, Bien vengas, mal, si vienes solo, p. 614.

15 Calderón, Bien vengas, mal, si vienes solo, p. 631.

16 Calderón, Bien vengas, mal, si vienes solo, p. 633. 
Y elige mentir a su padre, diciéndole que el escondido en su cuarto es don Juan, para encubrir así a su amado don Diego.

La densificación del enredo es tal que en la tercera jornada se encuentran los tres galanes y las dos damas en casa de D. Bernardo y es entonces cuando se produce el desenlace que, como es habitual, es rápido y un poco gratuito. Son escenas de «escondidos» tan frecuentes en las comedias de enredo de la época, hasta el punto de que un gracioso de otra comedia calderoniana alude a este tópico con el consiguiente comentario metateatral: «iQue sea fuerza / que luego escondites haya / al primer paso!» ${ }^{17}$.

La obra tiene un buen gracioso, Espinel, sobre el que descansa la comicidad de la misma, y una criada, Inés, que sirve de consejera a su dama, inmersa en numerosas dudas y tribulaciones para salvaguardar su honor, su amor y el secreto que debe guardar para no traicionar a su amiga. Ante los lances y situaciones que se suceden los personajes utilizan con frecuencia los comentarios metateatrales, tal y como es habitual en esta primera etapa del teatro calderoniano:

ANA

Deso mismo

he visto yo una comedia.

ANA

Si esto acaso se escuchara

en una farsa, ¿faltara

quien dijese que no había

sido posible causar

tantas cosas un sujeto ${ }^{18}$

Pero este tipo de alusiones aparecen sobre todo en boca de los criados, Espinel e Inés:

ESPINEL

¡Válgame Dios! ¿Es tramoya?

ESPINEL
Mosqueteros de la paz,
árbitros de la comedia
todos somos de la carda
y a todos pido clemencia.

17 Calderón, Cada uno para sí, pp. 181-182. Para las características y rasgos principales de la comedia de capa y espada, ver Arellano, 1999, pp. 37-69.

18 Calderón, Bien vengas, mal, si vienes solo, pp. 617 y 619. 
INÉS

Estaba embozado un hombre en la calle... ¡Mal hubiesen las comedias, que enseñaron engaños tan aparentes! ${ }^{19}$

\section{TrAYECTORIA ESCÉNICA}

Aparte de la fecha del estreno en palacio, el 16 de diciembre de 1635, pocos datos tenemos sobre la trayectoria escénica de esta comedia calderoniana en el siglo XVII. Sólo sabemos que se representó en la Montería de Sevilla en octubre de 1641 por Los Cobaleda ${ }^{20}$. Se trata de la compañía de Pedro Cobaleda, Francisco Vélez de Guevara y Francisco Álvarez de Vitoria, que representó en la Montería entre el 21 de septiembre y el 10 de diciembre ${ }^{21}$.

En cambio, los datos que tenemos para el siglo XVIII son mucho más ricos. Esta repentina popularidad quizá tenga que ver con la inclusión del texto en la edición de Vera Tassis y con las sueltas que se publicaron en dicho siglo. La comedia se representó con bastante asiduidad:

- una representación en Valencia entre 1716 y $1744^{22}$;

- cuatro representaciones en Sevilla en la calle de San Eloy entre 1776 y $1778^{23}$ y otras siete entre 1800 y $1820^{24}$;

— dos representaciones registradas en Barcelona en 1775 y $1778^{25}$.

- treinta y una representaciones en Madrid entre 1748 y 1808, 18 en el Príncipe y 13 en el teatro de la Cruz, según los datos que nos proporciona la cartelera de Andioc y Coulon ${ }^{26}$.

19 Calderón, Bien vengas, mal, si vienes solo, pp. 626, 621 y 631.

20 Sentaurens, 1984, p. 1096.

21 DICAT, 2008, «Pedro Cobalera».

22 Juliá, 1933, p. 122.

23 Aguilar Piñal, 1974.

${ }^{24}$ Las fechas exactas son las siguientes: 10-VII-1800, 1-V-1807, 8-IX-1810, 2-VI1812, 18-V-1813, 4-VII-1817 y 4-II-1820 (Aguilar Piñal, 1968, p. 18).

25 Par, 1929, pp. 337 y 341. Cita además una representación de una obra titulada Venga mal, que muy bien podría ser la misma, el 4 de diciembre de 1730 por la compañía de Nicolás Moro (Par, 1929, p. 333).

26 Andioc y Coulon, 1996, p. 639. 
- los días 22 y 23 de octubre de 1811 vuelve a representarse esta obra en el teatro del Príncipe por la compañía de Isidoro Máiquez ${ }^{27}$. Precisamente se conserva en la Biblioteca Histórica de Madrid (BHM, Tea 1-10-7) el apunte que sirvió de base para esta representación con la aprobación firmada el 26 de septiembre. También se alude a alguno de los actores del reparto:
Cas. $^{\text {va }}$
D. Luis, galán
Avec $^{a}$
D. Juan de Lara, galán
G. ${ }^{n}$
D. Diego de Silva, galán.

Las dos primeras abreviaturas corresponden a Santiago Casanova y Bernardo Avecilla, actores de la compañía de Isidoro Máiquez por esas fechas ${ }^{28}$.

- todavía el 1 de mayo de 1813 se anunciaba una representación de la obra de Calderón en Cádiz en el periódico El conciso ${ }^{29}$

\section{LA REFundición de Gorostiza: TAMBIÉN HAY SECRETO EN MUJER}

Del primer tercio del siglo XIX data la refundición de Bien vengas, mal, si vienes solo que llevó a cabo el dramaturgo mexicano Manuel Eduardo de Gorostiza. Probablemente se produjo de una manera un tanto casual, ya que la idea surgió al hilo de una conversación con unos amigos, tal y como se explica en la advertencia inicial a la edición de esta obra en 1826. Es una incógnita las razones que llevaron a sus amigos a elegir esta comedia de Calderón para que el escritor mexicano hiciera su arreglo. Quizá algo tuvo que ver el éxito que por aquellas fechas todavía tenía la obra calderoniana en los escenarios madrileños y sevillanos. El 22 de octubre de 1811 había sido representada en el Príncipe por el gran Isidoro Máiquez. La elección de este título también pudo deberse a la búsqueda de una obra de complicado enredo que permitiera mostrar a Gorostiza todo su ingenio.

27 Cotarelo, 2009, p. 682.

28 Cotarelo, 2009, p. 537.

${ }^{29}$ Ver Hemeroteca Digital de la Biblioteca Nacional de España. 
Manuel Eduardo de Gorostiza ${ }^{30}$ había nacido en el puerto de Veracruz el 13 de octubre de 1789. A la muerte de su padre en 1794, su madre, la gaditana María del Rosario Cepeda, decidió regresar a España con sus hijos. Manuel Eduardo, tras abandonar la carrera eclesiástica, se dedicó a las armas, llegando a ser capitán de granaderos en 1808 y coronel en 1814. Después se retiró del ejército y se dedicó a la política y a las letras. En 1818 escribe su primera obra dramática, Indulgencia para todos, estrenada en septiembre por la compañía del teatro del Príncipe con la participación de Isidoro Máiquez.

En 1820 colaboró con Félix Mejía en El Constitucional. Correo General de Madrid y con el mismo Mejía y Francisco José Iznardi en la revista Cetro Constitucional. Ese mismo año se representa Don Dieguito y al año siguiente estrena Virtud y patriotismo o el primero de enero de 1820, para celebrar el aniversario de la revolución de Cádiz contra el absolutismo de Fernando VII, y Una noche de alarma en Madrid. En mayo de 1821 es cuando lleva a escena También hay secreto en mujer, título que recibe la refundición de la comedia calderoniana Bien vengas, mal, si vienes solo, y tuvo un total de 5 representaciones ${ }^{31}$. En 1822 se representa Lo que son mujeres, basada en la comedia de igual título de Rojas Zorrilla.

$\mathrm{Al}$ año siguiente es desterrado de España por Fernando VII y se asienta en Londres. En 1825 será nombrado cónsul general interino en Bélgica y en 1830 ministro plenipotenciario de México ante la corte británica. En 1833 vuelve a su país de origen y será nombrado bibliotecario nacional y miembro de la Comisión de Instrucción Pública. De esta época son Contigo pan y cebolla (1833) y Don Bonifacio (1835). En 1835 será nombrado director del Teatro Principal de México y seguirá escribiendo y representando obras suyas. A partir de entonces ocupará distintos cargos políticos hasta su muerte en Tacubaya el 23 de octubre de 1851.

La refundición de Bien vengas, mal, si vienes solo se publicó por primera vez en 1826 en la edición del teatro del mexicano que se hizo 1987.

30 Sobre el autor mexicano, ver Aguilar, 1932, María y Campos, 1959, y Ortuño,

31 Adams, 1936, p. 354. En el Nuevo Diario de Madrid aparece ya como «repartida para ponerse en ensayo» los días 9 y 11 de mayo de ese año. En el Diario de Madrid del 20 de mayo aparece "en lista para ejecutar inmediatamente» (ver Hemeroteca digital de la Biblioteca Nacional de España). 
en París ${ }^{32}$. A finales del siglo XIX se volvió a publicar en México ${ }^{33}$, formando parte de nuevo de la edición de sus obras. Precisamente, en la advertencia a la edición de 1826 señala el editor que Gorostiza era "enemigo de todo fanatismo incluso el literario» y se le había encargado al mexicano que «refundiese dos comedias a su modo y que las presentase luego para ser juzgadas». Las dos obras que elige son, pues, la comedia calderoniana de la que nos ocupamos, que arreglará con el título de También hay secreto en mujer, y Lo que son mujeres de Rojas Zorrilla, que adapta manteniendo el mismo título ${ }^{34}$. En ese prólogo se advierte que Gorostiza «refundiolas efectivamente, leyolas, gustaron, representáronse, aplaudiéronse y no se imprimieron hasta ahora». Además, las obras no han perdido nada «de su originalidad y picante, por haber quedado ambas de escena fija, y por estar sujetas a las otras unidades $»^{35}$.

En el caso de la obra de Calderón, la refundición de Gorostiza trata de someter la obra a las tres unidades, en especial en cuanto a la unidad de lugar, ya que como se señala en la advertencia la comedia es de escena fija. Todos los lances se suceden en la casa de D. Bernardo donde entran una y otra vez los distintos personajes. Todo transcurre en un día, desde de la primera entrevista entre las dos amigas a primera hora de la mañana hasta el desenlace que se produce esa misma noche. El refundidor modifica la división en jornadas: la tercera jornada se divide en dos, con lo cual de las tres iniciales pasamos ahora a cuatro actos. No le gusta tampoco el título al dramaturgo mexicano y lo cambia por uno que, sin embargo, mantiene el aire calderoniano (También hay secreto en mujer). Calderón había utilizado para el título de la comedia un refrán bien conocido, que aparece en El Quijote en boca de Sancho (Quijote, II, 55), y es aludido, como es habitual, en

32 También hay secreto en mujer, en Apéndice al Teatro escogido de Manuel Eduardo de Gorostiza, París, en casa de Rosa y Cia., Libreros, calle de Chartres, ním. 12, 1826, tomo I, pp. [1]-208.

33 Obras, México, Imprenta de Victoriano Agüeros, 1899 (Biblioteca de Autores mexicanos, 26), III, pp. 1-156.

${ }^{34}$ Sobre la refundición de esta obra ya hemos hablado en otro lugar: González Cañal, 2007.

35 Gorostiza, «Advertencia», en Apéndice al Teatro escogido..., h. i-iv. Modernizamos las grafias y la puntuación. 
los versos finales de la segunda y tercera jornada por el gracioso Espinel.

La representación de mayo de 1821 tuvo una reseña en El Censor que es digna de tener en cuenta. El autor de la reseña comienza señalando que esta obra es inferior a la ya citada También hay duelo en las damas, sobre todo porque la intriga tiene menos solidez y no está bien trabada. Critica en Bien vengas, mal... que el secreto que guarda doña Ana, causante de los celos de don Diego - los papeles amorosos y el retrato que le ha confiado su amiga doña María-, no parece obligar con tanta fuerza a la dama como para poner en peligro su propia relación amorosa.

De la refundición de Gorostiza señala que ha eliminado dos escenas, la primera de ellas con razón, por ser «muy ridícula y sin resultados dramáticos ${ }^{36}$. Se trata de la primera escena nocturna con la que se abre la obra en la que aparece D. Luis con su criado Guzmán, personaje que el mexicano suprime, que van a presenciar las cuchilladas a la puerta de la casa de doña María entre D. Juan y otro caballero que finalmente cae muerto. Espinel que estaba escondido sale después y D. Luis y Guzmán le interrogan. Todo este cuadro, escrito en redondillas, es eliminado en la refundición para lograr que todo ocurra en un único lugar y acortar el tiempo en que se desarrollan los hechos. En total 236 versos suprimidos. En el segundo cuadro, que se desarrolla en casa de don Bernardo, están de conversación doña Ana y la criada Inés. Así comienza la obra de Gorostiza, aunque hasta la llegada de doña Ana los versos son de su propia cosecha y no de Calderón, entre otras cosas porque el dramaturgo áureo usa redondillas y el escritor mexicano utiliza un romance en -e. En ese rápido diálogo, Gorostiza presenta las inquietudes amorosas de doña Ana: desdeña a don Luis, hermano de su amiga y ama a don Diego. Con la entrada de doña María, comienza la escena II de Gorostiza y es en el relato que hace entonces doña María cuando la correspondencia entre los dos textos comienza. Los primeros 336 versos calderonianos han quedado reducidos a 94 versos en la adaptación del mexicano.

En cambio, la reseña de El Censor critica la supresión del conceptuoso soneto en que estaba envuelto el retrato que doña María con-

36 El Censor, 1821, p. 180. 
fía a su amiga. El autor de la reseña dice que «no debió haberse suprimido». Es el siguiente:

Cuando sutil pincel me repetía, yo en vos, hermoso dueño, imaginaba, y tanto en vos mi amor me transformaba, que en vos el alma más que en mí vivía.

Y así, cuando volver quiso a la mía, ya en dos mitades dividida estaba, y ella entre dos semblantes ignoraba a cuál de aquellos dos asistiría.

Así el retrato, a quien el alma muestro partiéndole mi amante desvarío, por parecerse mío, va a ser vuestro,

y por ser vuestro, ya parece mío; porque el pincel le iluminó tan diestro, que retrató también el albedrío ${ }^{37}$.

También critica la supresión del monólogo de D. Diego al principio de la segunda jornada, "porque pinta muy bien la lucha entre el amor y el enojo». En efecto, estas dudas y vacilaciones del galán preparan la escena de reconciliación entre los dos enamorados que viene a continuación, que, según este crítico, «perpetuará esta pieza en nuestro teatro, mientras agraden el lenguaje, los buenos versos y la verdad de las pasiones». Más adelante señala sobre este pasaje:

en su género es quizá la mejor de nuestro antiguo teatro. Los esfuerzos de D. Diego para no quejarse, y las quejas que da al mismo tiempo, la seriedad burlona de doña Ana, la dignidad con que esta da sus satisfacciones, la alegría de su amante al recibirlas, y la sal y urbanidad del diálogo, hacen esta escena comparable a las mejores de Molière, cuando este profundo escritor de caracteres se complace en pintar las niñerías del amor ${ }^{38}$.

37 Calderón, Bien vengas, mal,..., p. 612. Gorostiza lo suprime aunque los personajes aluden a él en diálogos posteriores (Gorostiza, También hay secreto en mujer, pp. 172 y 179$)$.

38 El Censor, 1821, pp. 182-184. También elogia este pasaje Ángel Lasso de la Vega: «La escena de celos del acto segundo de Bien vengas mal, entre D. Diego y Dña. Ana, es una de las mejores de esta obra, agradable por su corte y la discreción con que ambos se expresan» (Lasso, 1881, p. 233). 
Aparte de Guzmán, criado de D. Luis, también elimina Gorostiza la criada Juana, que tiene muy pocas intervenciones en la comedia calderoniana y sólo sirve para acompañar a su ama en sus idas y venidas.

Pero no acaban aquí los cambios: hay varios monólogos reelaborados y una menor presencia del personaje de D. Luis, mientras que se da un mayor protagonismo a la figura del padre, D. Bernardo. Además, el desenlace es semejante en ambas obras, pero en el caso de la refundición es el personaje de D. Juan el que se interpone entre los dos pretendientes de doña Ana pidiendo al padre que deje elegir a su hija. Los versos finales que en Calderón aluden al título de la comedia, con el consiguiente chiste sobre el matrimonio, no son demasiado afortunados en Gorostiza; en ellos pretende justificar además el nuevo título elegido:

\section{Calderón}

ESPINEL

Ahora entro yo con Inés, porque vean desta suerte que no viene solo un mal, pues tantos juntos nos vienen el día que nos casamos. Perdonen vuestras mercedes ${ }^{39}$.

\section{Gorostiza}

ESPINEL

Alto aquí, y nadie me chiste, porque en término tan breve, es dificil demostrar mejor, ni más claramente, que el secreto en la mujer es posible.

YNÉS

Ciertamente.

Mas pues el nuestro causó tantos dimes y diretes, casi, casi me dan ganas de pedir a las mujeres, que no nos imiten.

ESPINEL

¿Sí?

Pues concedido lo tienes ${ }^{40}$.

39 Calderón, Bien vengas, mal,..., p. 634.

40 Gorostiza, También hay secreto en mujer, p. 208. 
Además, Gorostiza peina el texto de Calderón y lo somete a numerosos retoques. Por una parte, procura siempre romper los monólogos o largos parlamentos de los personajes calderonianos, transformándolos en diálogos de réplicas ágiles y breves. Así ocurre en el relato que le hace D. Juan a D. Bernardo en el primer acto y en la réplica de este ${ }^{41}$. Por otra parte, sustituye a menudo palabras y expresiones por sinónimos o giros que considera más adecuados. Véase, en este sentido, los siguientes versos que condensan el dilema que vive doña Ana:

\section{Calderón \\ GorostizA}

ANA

(Si suceden

dos daños, es el menor

el que ha de elegirse siempre).
DOÑA ANA

Si suceden

dos males, siempre el menor ha de elegir el prudente ${ }^{42}$.

En general, no estuvo demasiado afortunado el autor de Indulgencia para todos en esta refundición de la comedia áurea. La obra de Calderón queda desdibujada en esta versión y los cambios y añadidos no mejoran ni la intriga ni el estilo del original. No es de extrañar que este experimento se olvidara con celeridad mientras, por el contrario, las obras originales del dramaturgo mexicano tuvieron cierto éxito en los teatros españoles durante décadas.

\section{La refundición de Ángel María Dacarrete}

Ya avanzado el siglo XIX encontramos una nueva refundición de la comedia calderoniana a cargo de Ángel María Dacarrete (1827-1904), médico, académico y autor de una interesante obra dramática. La obra fue publicada en Madrid en la Imprenta de José Cuesta 1854 y luego en 1861, siendo estrenada en el teatro del Príncipe el 17 de enero de 1865, con Teodora Lamadrid en el papel principal. La obra está

${ }^{41}$ Calderón, Bien vengas, mal,..., p. 610; y Gorostiza, También hay secreto en mujer, pp. 29-32.

42 Calderón, Bien vengas, mal,..., p. 633; y Gorostiza, También hay secreto en mujer, p. 200 . 
«refundida y acomodada a la escena moderna en cuatro actos», tal y como reza la portada. Los actos están divididos en numerosas escenas con largas acotaciones introductorias para situar la acción. Lo más destacable es que al principio de la obra el dramaturgo decimonónico otorga un papel al personaje de don Fadrique de Silva, galán al que Calderón tan solo alude como un caballero que cae muerto en la primera escena. Esta refundición se abre con una primera escena en la que este caballero tiene una serie de intervenciones hasta que muere en el enfrentamiento nocturno con don Juan, a la puerta de la casa de su dama, en la décima escena.

A pesar del estreno en Madrid en 1865, tampoco tuvo demasiada repercusión esta arreglo de Dacarrete del texto calderoniano.

\section{EL OLVIDO FINAL}

Tras estas dos refundiciones, la comedia de Calderón cae definitivamente en el olvido. No ha vuelto a aparecer en los escenarios y tampoco ha tenido demasiada repercusión en la crítica. Sin embargo, como hemos visto, se trata de una comedia de enredo bien construi$\mathrm{da}$, con una intriga que mantiene el interés hasta el final y con algunos detalles dignos de tener en cuenta. Por ello gozó de cierto atractivo y popularidad en la cartelera española durante cerca de un siglo. En este caso probablemente sean las refundiciones decimonónicas las que hicieron un flaco favor a la obra original, hasta el punto de hacerla desaparecer definitivamente del repertorio dramático nacional. 


\section{Bibliografía}

Adams, N. B., «Siglo de Oro plays in Madrid, 1820-1850», Hispanic Review, 4, 1936, pp. 342-357.

Aguilar, M. E., Estudio bio-bibliográfico de don Manuel Eduardo de Gorostiza, México, Universidad Nacional de México, 1932.

Aguilar Piñal, F., Cartelera prerromántica sevillana. Años 1800-1836, Madrid, CSIC, 1968 ("Cuadernos Bibliográficos», XXII).

- Sevilla y el teatro en el siglo XVIII, Oviedo, Cátedra Feijoo, Facultad de Filosofia y Letras, Universidad de Oviedo, 1974.

Andioc, R., y M. Coulon, Cartelera teatral madrileña del siglo XVIII (17081808), Toulouse, Presses Universitaires du Mirail, 1996, 2 vols.

Arellano, I., «La comedia de capa y espada. Convenciones y rasgos genéricos», Cuadernos de teatro clásico, 1, 1988, pp. 27-49, recogido en Convención y recepción. Estudios sobre el teatro del Siglo de Oro, Madrid, Gredos, 1999, pp. 37-69.

Calderón de la Barca, P., Bien vengas, mal, si vienes solo, en Obras completas, ed. Á. Valbuena Briones, Madrid, Aguilar, 1973, II, pp. 600-634.

- Cada uno para sí, ed. J. M. Ruano de la Haza, Kassel, Reichenberger, 1982.

Cotarelo y Mori, E., Ensayo sobre la vida y obras de don Pedro Calderón de la Barca. Parte Primera, Madrid, Tipografía de la Revista de Archivos, Bibliotecas y Museos, 1924 [Rep. facsímil, Madrid / Frankfurt, Iberoamericana / Vervuert, 2001].

- Isidoro Máiquez y el teatro de su tiempo, estudio preliminar de J. Álvarez Barrientos, Madrid, Publicaciones de la Asociación de Directores de Escena, 2009.

Cruzada Villaamil, G., "Teatro antiguo español. Datos inéditos que dan a conocer la cronología de las comedias representadas en el reino de Felipe IV, en los Sitios Reales, el Alcázar de Madrid, Buen Retiro y otras partes, sacados de los libros de gastos y cuadernos de nóminas de aquella época que se conservan en el Palacio de Madrid», El Averiguador, I, Madrid, Rivadeneira, 1871, pp. 7a-11b, 25b-27a, 73a-75a, 106a-108a, 123b-125a, 170a-172a, 201b-202b.

Diccionario biográfico del teatro clásico español (DICAT). Edición digital, dir. T. Ferrer Valls, Kassel, Reichenberger, 2008.

El Censor, periódico político y literario, Madrid, en la imprenta del Censor, por D. León Amarita, 1821, IX, pp. 177-186.

González CAÑAL, R., "Lo que son mujeres en los escenarios», Revista de Literatura, LXIX, núm. 137, enero-junio 2007, pp. 109-124.

Gorostiza, M. E. de, También hay secreto en mujer, en Apéndice al Teatro escogido de Manuel Eduardo de Gorostiza, París, en casa de Rosa y Cia. Libreros, calle de Chartres núm. 12, 1826, tomo I, pp. [1]-208. 
Hartzenbusch, J. E. (ed.), Obras de don Pedro Calderón de la Barca, I, Madrid, Rivadeneyra, 1848 (BAE, VII).

Juliá Martínez, E., «Preferencias teatrales del público valenciano en el siglo XVIII», Revista de Filología Española, 20, 1933, pp. 113-159.

Lasso de la Vega, Á., Calderón de la Barca. Estudio de las obras de este insigne poeta, Madrid, Imprenta y fundición de M. Tello, 1881.

María y Campos, A. de, Manuel Eduardo de Gorostiza y su tiempo. Su vida. Su obra, México, Talleres Gráficos de la Nación, 1959.

Ortuño Martínez, M., Manuel Eduardo de Gorostiza, hispano-mexicano, romántico y liberal, Madrid, Ayuntamiento de Madrid, 1987.

PAR, A., "Representaciones teatrales en Barcelona durante el siglo XVIII", Boletín de la Real Academia Española, 16, 1929, pp. 326-349, 492-513 y 594-614.

Reichenberger, K. y R., Bibliographisches Handbuch der Calderón Forschung / Manual Bibliográfico Calderoniano, Kassel, Thiele und Schwarz, 1979-2009, t. I-IV.

Rennert, H. A., "Notes on the Chronology of the Spanish Drama», Modern Language Review, 2, 1906-1907, pp. 331-341 y III, 1907-1908, pp. 43-55.

Sentaurens, J., Seville et le théâtre. De la fin du Moyen Âge à la fin du XVII siècle, Bordeaux / Lille, Université de Bordeaux / Atelier, 1984, 2 vols.

Serralta, F., "También hay duelo en las damas: Calderón y Solís», Criticón, 38, 1987, pp. 100-111.

— «El tipo del "galán suelto": del enredo al figurón», Cuadernos de Teatro Clásico, 1, 1988, pp. 83-93.

Shergold, N. D. y J. E. VArey, «Some early Calderón dates», Bulletin of Hispanic Studies, 38, 1961, pp. 274-286.

Wilson, E. M., «An Early List of Calderón's comedias», Modern Philology, 60, 1962, pp. 95-102. 